Revue musicale OICRM

\title{
Orienter l'écoute et la réception. La diffusion des préfaces aux poèmes symphoniques de Liszt dans les salles et la presse en Allemagne et en France (1850-1914) : une enquête préliminaire
}

\section{Céline Carenco et Nicolas Dufetel}

Volume 7, numéro 1, 2020

Écrits de compositeurs et espaces médiatiques

URI : https://id.erudit.org/iderudit/1069470ar

DOI : https://doi.org/10.7202/1069470ar

Aller au sommaire du numéro

Éditeur(s)

Observatoire interdisciplinaire de création et recherche en musique (OICRM)

ISSN

2368-7061 (numérique)

Découvrir la revue

Citer cet article

Carenco, C. \& Dufetel, N. (2020). Orienter l'écoute et la réception. La diffusion des préfaces aux poèmes symphoniques de Liszt dans les salles et la presse en Allemagne et en France (1850-1914) : une enquête préliminaire. Revue musicale OICRM, 7(1), 36-61. https://doi.org/10.7202/1069470ar
Résumé de l'article

Connu comme l'inventeur du " poème symphonique ", Liszt entoure un grand nombre de ses oeuvres d'écrits aux formes diverses, que nous appelons "paratextes". Ces paratextes semblent constituer un puissant moyen de médiatiser sa musique, mais qu'en est-il réellement de leur diffusion, de leur circulation et de leur réception ? Nous partirons à la recherche des textes de préface des poèmes symphoniques de Liszt, de leurs traces et de leur influence dans la presse et plus largement dans l'espace médiatique français et allemand (incluant les affiches, programmes, annonces et comptes rendus de concert), afin d'interroger leur fonction dans la médiatisation de sa musique : dans quelle mesure les paratextes permettent-ils au compositeur d'orienter l'écoute ? Et quels rôles jouent-ils dans la réception de sa musique ? Après quelques considérations sur le rôle des paratextes dans l'esthétique lisztienne, nous proposerons quelques pistes de recherche à travers plusieurs études de cas (exemples de diffusions en Allemagne, à, Paris, Angers et Nancy). 


\title{
Orienter l'écoute et la réception. La diffusion des préfaces aux poèmes symphoniques de Liszt dans les salles et la presse en Allemagne et en France (1850-1914) : une enquête préliminaire
}

\author{
Céline Carenco et Nicolas Dufetel
}

\begin{abstract}
Résumé
Connu comme l'inventeur du "poème symphonique ", Liszt entoure un grand nombre de ses œuvres d'écrits aux formes diverses, que nous appelons "paratextes ». Ces paratextes semblent constituer un puissant moyen de médiatiser sa musique, mais qu'en est-il réellement de leur diffusion, de leur circulation et de leur réception? Nous partirons à la recherche des textes de préface des poèmes symphoniques de Liszt, de leurs traces et de leur influence dans la presse et plus largement dans l'espace médiatique français et allemand (incluant les affiches, programmes, annonces et comptes rendus de concert), afin d'interroger leur fonction dans la médiatisation de sa musique : dans quelle mesure les paratextes permettent-ils au compositeur d'orienter l'écoute ? Et quels rôles jouent-ils dans la réception de sa musique? Après quelques considérations sur le rôle des paratextes dans l'esthétique lisztienne, nous proposerons quelques pistes de recherche à travers plusieurs études de cas (exemples de diffusions en Allemagne, à, Paris, Angers et Nancy).
\end{abstract}

Mots clés : concert ; Franz Liszt ; médiation; musique à programme; presse.

\begin{abstract}
Known as the inventor of the "symphonic poem," Liszt accompanies many of his works with writings of various forms, which we call "paratexts." These paratexts seem to be a powerful way to get media attention on his music; but what can be said about their distribution, circulation, and reception? We will look for the prefaces of Liszt's symphonic poems, their traces and their influence in the press and more broadly in the French and German media space (including posters, programs, announcements and concert reports), in order to question their function in the media coverage of his music: to what extent do the paratexts allow the composer to orientate the listening process? What roles do they play in music reception? After some considerations on the role of paratexts in the Lisztian aesthetics, we propose some new ideas through several case studies (diffusion in Germany, Paris, Angers, and Nancy).

Keywords: concert; Franz Liszt; mediation; press; program music.
\end{abstract}


Défini comme un écrit du compositeur entourant sa partition, le paratexte auctorial constitue à la fois un moyen de médiation de la musique par les mots et un outil de médiatisation de l'œuvre musicale à laquelle il est attaché ${ }^{1}$. Repris lors de la diffusion de l'œuvre, qu'il soit imprimé sur des programmes de salle distribués au concert ou réutilisé sur des affiches ou dans des comptes rendus de presse, il en fait la promotion. L'utilisation d'un paratexte permet ainsi au compositeur de créer un premier espace médiatique autour de l'œuvre, grâce auquel il contrôle dans une certaine mesure son insertion dans un espace médiatique plus large.

L'usage des paratextes touche toutes les périodes de l'histoire de la musique, mais prend une importance particulière au XIX ${ }^{\mathrm{e}}$ siècle avec le développement de ce qu'on a appelé la "musique à programme ", dans laquelle des textes (souvent appelés " préfaces » ou " programmes ») accompagnent des partitions instrumentales. Par bien des aspects, lorsqu'ils sont imprimés et distribués dans les salles de concert, les textes des préfaces des poèmes symphoniques de Liszt préfigurent les notices de programme encore fournies de nos jours aux auditeurs comme « documents d'accompagnement du concert » (Donin 2011, Couture 2015). Une lecture suivie des feuillets distribués au public des concerts symphoniques des années 1830 jusqu'au début du $\mathrm{XX}^{\mathrm{e}}$ siècle donne ainsi à voir l'élaboration progressive des conventions rédactionnelles de ce type de document, leur diversité et leur évolution selon les genres et les milieux musicaux. Un premier aperçu de l'ensemble de ce vaste corpus, à partir de l'exemple de la musique à programme lisztienne, laisse apparaître une tendance croissante à s'écarter d'un discours uniquement auctorial pour laisser de plus en plus de place à la plume du musicographe, du critique et du musicologue lorsque le propos devient analytique $^{2}$. L'écrit initial du compositeur reste cependant présent dans la majorité des cas, qu'il soit rigoureusement cité ou simplement rappelé de manière plus évasive à travers le choix des mots employés.

Pourtant la réalité de la diffusion concrète des paratextes lisztiens auprès du public doit être interrogée et inscrite dans la réception assez peu étudiée de la musique symphonique de Liszt jusque dans la première moitié $d u x^{e}$ siècle (en particulier en France) : cette diffusion peut être systématique, ou au contraire assez rare, ou même dans certains cas inexistante.

La traque des paratextes de Liszt, de leurs traces et de leurs utilisations dans la presse et plus largement dans l'espace médiatique français et allemand (incluant les notices de programme, les affiches, annonces et comptes rendus de concert), permettrait d'une part d'évaluer concrètement leur degré de diffusion. D'autre part, elle inviterait à interroger leur fonction dans la médiatisation de la musique de Liszt et leur rôle de médiation auprès du public : au-delà de la dimension esthétique, la rédaction de paratextes par le compositeur a-t-elle pour but concret de contrôler la

1 Sur la notion de paratexte, voir Genette 1987 ; sur son application à la musique, voir Escal 1996, et aux écrits de Liszt, Dufetel 2013.

2 Certaines pages de Michel Dimitri Calvocoressi sur Liszt sont ainsi utilisées dès 1911 sur des notices de programme distribuées en concert, notamment à Nancy. 
diffusion et l'écoute de sa musique ? Si oui, dans quelle mesure le permettent-ils ? Ont-ils le pouvoir d'influencer non seulement l'interprète, mais aussi l'auditeur, à travers l'induction d'une attitude d'écoute particulière?

Devant l'immensité du champ offert par un corpus très large, nous avons choisi de mener une enquête préliminaire qui procède par sondages dans certains fonds précis, sans prétention à l'exhaustivité. Après quelques incursions en Allemagne, nous avons observé la situation lors de l'introduction des poèmes symphoniques en France, à Paris et en province dans deux sociétés de concert particulièrement actives au tournant du siècle : la Société des concerts populaires d'Angers ( $\mathrm{SCP}$ ) fondée en 1877, et les concerts développés sur le même modèle par Guy Ropartz au conservatoire de Nancy à partir de 1894. Avant d'entrer dans ces données concrètes, il apparaît cependant nécessaire d'apporter quelques précisions sur le rôle du programme dans l'esthétique lisztienne.

LE RÔLE DU PROGRAMME CHEZ LISZT

Le modèle de la Symphonie fantastique

Berlioz appelle " programme » le texte qu'il rédige et joint à la Symphonie fantastique dès 1830. Geste auctorial fort: il a lui-même fait imprimerle programme dela Fantastique au Figaro avant la première exécution publique de la symphonie le 5 décembre ${ }^{3}$. Certes, la pratique de rédiger un programme littéraire pour une symphonie n'est pas entièrement nouvelle, puisque la conception berliozienne du " genre instrumental expressif ${ }^{4}$ » prend racine notamment dans la musique de Beethoven. Cette pratique connaîtra d'ailleurs chez Berlioz diverses modalités au-delà de l'exemple de la Symphonie fantastique : dès sa deuxième symphonie inspirée entre autres par le Childe Harold de Byron, Berlioz abandonne l'idée d'un programme entièrement rédigé au profit de simples titres évocateurs attribués à chacun des mouvements ${ }^{5}$. Mais en 1830, 1'idée d'un texte rédigé associé à une symphonie apparaît clairement comme une nouveauté pour les Parisiens : ils soulignent les aspects littéraires et narratifs du programme et posent immédiatement la question du sens précis que peut avoir la musique instrumentale ${ }^{6}$.

3 Voir [Berlioz] 1830, mais également, à ce sujet, Berlioz 1972 et Ramaut 2018.

4 Berlioz (1830), "Aperçu sur la musique classique et la musique romantique ", Le Correspondant, 22 octobre, dans Gérard et Cohen 1996, p. 63-68.

5 Les titres de chacun des mouvements d'Harold en Italie en dessinent ainsi le programme : « Harold aux Montagnes. Scènes de mélancolie, de bonheur et de joie ", "Marche de pèlerins chantant la prière du soir », "Sérénade d'un Montagnard des Abbruzes à sa maîtresse », " Orgie de Brigands. Souvenir des scènes précédentes ». Voir Bonds 1992 et Carenco 2013. En fait chacune des œuvres symphoniques de Berlioz expérimente un nouveau rapport de la musique au programme : il expérimente les monologues parlés dans Le retour à la vie, imagine pour Roméo et Juliette une "Symphonie dramatique avec chœurs », etc.

6 "Voici la première fois qu'on s'est avisé de donner un sens précis à la musique instrumentale » (Anonyme 1830). 
C'est en effet un enjeu central : les défenseurs de la musique à programme (qui se regrouperont dans les années 1850 autour de Franz Liszt et Franz Brendel) s'appuient sur une esthétique de la musique instrumentale pensée comme le plus romantique de tous les arts, illustrée par les propos d'Hoffmann, au début du siècle, sur la $5^{e}$ symphonie de Beethoven ${ }^{7}$. Ils postulent donc non seulement le potentiel évocateur de l'art des sons, mais encore la capacité de la musique à dire l'indicible : pour eux, la question n'est pas de savoir si la musique peut évoquer quelque chose, mais plutôt de savoir dans quelle mesure elle peut signifier quelque chose de précis tout en tirant en même temps parti de sa capacité à exprimer le vague de ce qui se situe au-delà des mots (Candoni 2012, p. 163-209).

En pratique, l'association d'un texte à de la musique instrumentale dans la musique à programme s'effectue de deux façons : lors de l'exécution de l'œuvre et lors de la publication de la partition.

Lors de l'exécution de l'œuvre, le texte du programme peut être diffusé en amont par le biais de la presse et parfois des affiches, et il peut être directement imprimé et distribué à l'auditoire dans la salle. L'habitude de distribuer au spectateur un feuillet ou un livret lors de l'exécution d'une œuvre musicale est cependant antérieure au $\mathrm{XIX}^{\mathrm{e}}$ siècle. En dehors de l'opéra dont le livret peut bien entendu constituer un support textuel utilisé avant ou pendant l'audition, cet usage est déjà présent au concert au $\mathrm{XVIII}^{\mathrm{e}}$ siècle, comme le montrent par exemple de récents travaux sur les « paroles du concert » dans plusieurs villes françaises (Doé de Maindreville 2015). Ces «paroles du concert » concernent toutefois avant tout la musique vocale. La nouveauté du geste berliozien consiste à associer la distribution d'un programme de salle à de la musique instrumentale, donnant l'idée que la musique contient ou exprime une intrigue comme à l'opéra ${ }^{8}$. Fournir les paroles d'œuvres vocales et distribuer un programme accompagnant une œuvre instrumentale relèvent bien entendu de considérations esthétiques très différentes, mais le geste concret, auquel le public peut être habitué, de recevoir un support textuel lors d'un concert, demeure l'élément important, même si Berlioz a changé d'avis au cours de sa carrière sur la nécessité de distribuer systématiquement le programme de la Fantastique à l'auditoire (Temperley 1971, Reynaud 2003). Présent lors des exécutions de la Symphonie fantastique à Paris le 5 décembre 1830, puis les 9 et 30 décembre 1832, et après avoir transcrit l'œuvre pour piano en 1833-1834, Liszt s'empare de cette idée. Il associe à ses œuvres symphoniques des textes plus ou moins longs, qui tout en s'inspirant du geste du compositeur français tendront à s'écarter, dans le mode de rédaction et le contenu précis des textes de la conception berliozienne ${ }^{9}$.

$7 \quad$ Article publié en deux livraisons les 4 et 11 juillet 1810 dans l'Allgemeine Musikalische Zeitung (Hoffmann [1810]1985, p. 38-50).

8 Berlioz écrit d'ailleurs que son programme "doit être considéré comme le texte parlé d'un opéra ", voir [Berlioz] 1830.

9 Serge Gut remarque que les programmes de Liszt sont plus poétiques que narratifs ou descriptifs (Gut 1989, p. 349). 
Lors de la publication de la partition, le texte du programme est habituellement imprimé après la page de titre et avant la musique, en guise de préface. Le programme de la Symphonie fantastique figure ainsi dans la première édition en grande partition de la symphonie en 1845 , et on peut noter que Liszt accorde encore de l'importance à la présence de ce programme dans la partition de Berlioz en 1876 : il insiste auprès de l'éditeur de la seconde version de sa " partition de piano » de la Fantastique pour qu'y figure le texte du programme de Berlioz ${ }^{10}$. L'idée d'une alliance très intime entre la musique et la poésie reste effectivement au cœur de la conception de Liszt et va se décliner dans ses œuvres de diverses manières.

\section{Le rôle des paratextes dans l'" union entre poésie et musique»}

La musique de Liszt, comme celle de ses contemporains, Berlioz et Schumann par exemple, entretient une relation particulière à la recherche $d^{\prime}$ ' alliance » entre musique et poésie, par l'intermédiaire des mots ${ }^{11}$. S'il s'agit d'une problématique commune à ces compositeurs, à leur génération et à l'esprit du temps, leurs propositions et leurs réponses à la question de la "poetische Musik" sont différentes. Chez Liszt, lecteur boulimique et bibliophile dès sa jeunesse, les mots sont omniprésents. Sa correspondance est remplie de références à ses lectures, et ses esquisses musicales, dès les années 1830, sont intimement liées à la littérature. On trouve en effet souvent des mots au cœur du processus génétique d'œuvres qui ne furent pas toutes achevées et publiées (Dufetel 2015, 2018). Dans ses partitions imprimées, pour résumer, ils se trouvent sous différentes formes qui sont autant de catégories de paratextes participant à la « constellation » programmatique de chaque œuvre. Les différents types de paratextes, chez Liszt, correspondent aux catégories suivantes :

1. Des titres : ils sont en eux-mêmes une sorte de programme; par exemple la pièce pour piano Lyon, liée à la révolte des canuts, ou les poèmes symphoniques Festklänge et Hamlet;

2. Des épigraphes : fragments placés au seuil de l'œuvre, comme dans Lyon (devise des canuts : "Vivre en travaillant ou mourir en combattant »), l'oratorio Christus, où un verset de Saint-Paul résume le cœur de la foi catholique du compositeur, et toutes les pièces de la première Année de pèlerinage, où ils complètent aussi le titre programmatique ; il faut absolument remarquer que dans l'édition originale de ces pièces, les épigraphes sont insérées dans les images, ce qui participe à la création d'une œuvre totale incluant, chez Liszt, musique, littéraire et art visuel par le médium de la partition (Dufetel 2015);

3. Des titres-citations : il s'agit d'une situation intermédiaire entre les deux premières catégories, c'est-à-dire de titres qui auraient pu être des épigraphes, ainsi Sunt Lacrymae rerum d'après Virgile (troisième Année de pèlerinage);

10 Voir la lettre de Liszt à Constantin Sander, le 15 novembre 1876 (Short 2003, p. 345-346). Le directeur de la maison Leuckart insèrera dans la réédition de la partition de piano de la Symphonie fantastique en 1877 la version du programme de 1845 .

11 Liszt écrit à Agnes Street-Klindworth, le 16 novembre 1860, vouloir réaliser une " grande idée » : le « renouvellement de la Musique par son alliance plus intime avec la Poésie » (Pocknell 2000, p. 352). 
4. Des citations au fil de la partition : placées à des moments particuliers, elles revêtent ainsi non pas un sens sur l'œuvre intégrale, mais une dimension épiphanique. Par exemple dans Die Ideale, où chaque section est accompagnée de strophes de la poésie de Schiller, dont Liszt change l'agencement, dans Les jeux d'eaux à la Villa d'Este où une citation de saint Jean évoque l'eau régénératrice (du baptême), et dans Christus, où des versets de l'Évangile de saint Matthieu marquent l'apparition de l'étoile et l'offrande des cadeaux par les rois mages ;

5. Des mots intégrés à la mélodie : ils pourraient être des épigraphes, mais ils ont été transformés, absorbés par la musique, car leurs syllabes induisent les contours de la mélodie. Par exemple, la devise des canuts de Lyon et le début de la DanteSymphonie, où les vers de Dante sont inscrits sous les notes des instruments ;

6. Des préfaces plus ou moins longues : on peut les considérer comme un développement, ou une "inflation » de l'épigraphie. Ce sont ces textes que l'on considère comme les « programmes».

À cet ensemble il faut ajouter des textes de natures diverses, qui ne sont pas des programmes à proprement parler, mais des moyens pour Liszt de communiquer avec ses interprètes, comme ses remarques générales sur la direction d'orchestre qu'il a rédigées pour ses œuvres symphoniques et qu'on trouve en tête de plusieurs éditions originales comme celles des poèmes symphoniques.

Enfin, on peut considérer l'ensemble des écrits de Liszt (réalisés avec l'aide de la comtesse d'Agoult ou de la princesse Wittgenstein) comme des paratextes à son œuvre musicale, chacun éclairant l'autre. À ce sujet, Liszt écrit dans son essai sur Robert Schumann, où il développe ses idées sur la critique, que les artistes doivent écrire eux-mêmes sur la musique, car ils le feront mieux que ceux qui ne la connaissent pas aussi bien qu'eux (Liszt 1882, p. 129-138).

Louis-Léon Gozlan, qui a publié en 1870 un Essai de critique musicale sur Liszt et ses poèmes symphoniques, lu et apprécié par le compositeur, écrit à propos de Prométhée que " pour une entière compréhension de l'œuvre nous renvoyons les artistes consciencieux à la préface explicative placée par le compositeur en tête de sa partition » (Gozlan 1870, p. 25). Le critique s'efface ici devant le compositeur. De façon générale, Gozlan considère que Liszt a dû faire précéder « quelques partitions de préfaces dans lesquelles l'idée philosophique est clairement développée et les mythes expliqués longuement ", parce que, écrit-il encore, le compositeur était dans la " presque certitude de rester incompris de la génération présente, même en Allemagne ». Il précise enfin que « le programme détaillé, destiné à guider l'auditeur et l'exécutant, ne saurait cependant suppléer à l'étude de l'œuvre en elle-même, lucide seulement pour les initiés » (ibid., p. 15).

Tous les textes de Liszt destinés à accompagner ses œuvres occupent des places différentes dans l'espace de médiation, et l'ensemble des catégories de paratextes forme leur constellation programmatique. Mais tous ne sont pas destinés à être connus du public : ainsi, les citations des catégories 2, 4 et 5 exposées ci-dessus sont-elles destinées au(x) seul(s) interprète(s). Le cas des préfaces et autres textes introductifs pour les poèmes symphoniques (catégorie 6) est différent : ils sont destinés à être connus du public, d'où notre interrogation concrète sur les modalités de leur médiation 
et diffusion. Liszt lui-même a demandé à ce que les textes soient diffusés, dans au moins un cas (première édition de Ce qu'on entend sur la montagne). Leur genèse et leur nature ne revêt aucune uniformité : il s'agit parfois de citations de poèmes plus ou moins longs (Hugo pour Ce qu'on entend sur la montagne, Hugo et Byron pour Mazeppa, par exemple, où leur association crée un intéressant exemple d'intertextualité). On ne peut affirmer avec certitude que Liszt soit seul l'auteur de chacun de ces textes, car il avait l'habitude de s'associer à la princesse Wittgenstein pour ses affaires littéraires. Le programme des Préludes, par exemple, n'est pas de Lamartine, comme on le croit souvent. Il s'agit d'un texte composite dont la genèse est difficile à cerner (voir Keym 2013, Bonner 1986 et Main 1979) : c'est une interprétation, une sorte de rétrodiction des Harmonies du poète, due à Liszt lui-même, peut-être avec l'aide de la princesse Wittgenstein. Haraszti accusait même cette dernière d'en être l'auteur et d'avoir forgé un faux texte lamartinien qu'il juge sévèrement et qui trahirait d'après lui l'esprit du poète et de la musique de Liszt (Haraszti 1953, p. 130-131). La question de la paternité des préfaces doit donc être approchée avec une grande prudence dans le cadre de ce que nous appellerons "l'atelier d'écriture de l'Altenburg ", comme le montre aussi l'exemple de la Dante-Symphonie dont la préface est signée par Richard Pohl (collaborateur littéraire de Liszt à Weimar dans les années 1850), mais abondamment révisée par la princesse et par le compositeur lui-même.

L'ordre de parution des poèmes symphoniques ne suit pas leur numérotation définitive. Ainsi, Ce qu'on entend sur la montagne, premier de la série, fut publié en 1857 après un premier ensemble comprenant Orphée et Les préludes (1856). C'est pourtant bien dans la première édition de cette partition que semble apparaître la consigne (en allemand et en français) : "Les lignes suivantes devront être toujours jointes au programme du concert, dans lequel ce poème symphonique sera exécuté " (Liszt [1857], s. p.). Suit, entre guillemets, un bref résumé programmatique du poème avec la citation de trois vers seulement. Les deux pages suivantes comportent les vers $\mathrm{du}$ poème, également en allemand et en français. Il semble donc que la consigne soit bien de distribuer le résumé et non le poème en lui-même. Ce texte, à l'image de celui pour Les préludes, est donc composite, ou une ébauche de glose : il mêle le poème à sa lecture par Liszt.

Ces courtes lignes sont vraisemblablement le seul exemple pour lequel Liszt demande une distribution au public, une pratique qui n'était pas encore généralisée en 1857. Il est possible également que l'on n'ait pas de traces documentaires des cas qui auraient existé. On sait par exemple que la Hof-Buchdruckerei de Weimar a publié une brochure de 30 pages intitulée Poèmes symphoniques de F. Liszt et comportant, sur deux colonnes en vis-à-vis, les préfaces en allemand et en français de Ce qu'on entend sur la montagne, Tasso, Les préludes, Orphée, Prométhée, Mazeppa, Héroïde funèbre ${ }^{12}$. Certains poèmes symphoniques ne comportent pas de programme, comme Hamlet

12 Voir par exemple les exemplaires conservés à Rochester University, Eastman School of Music (Sibley Music Library) et New York Public Library (Performing Arts Research Collections, Music Drexel 2403, sans Festklänge et Hungaria). Voir aussi J \& J Lubrano Music Antiquarians (2019), New York, Catalogue $\underline{n^{0}} \underline{85}$, septembre, lot $\mathrm{n}^{\circ} \underline{65}$. 
ou Von der Wiege bis zum Grabe : chacun est un cas particulier et le programme n'a pas toujours besoin d'être rédigé si le titre ouvre, peut-être dans la tradition des ouvertures de Beethoven, sur diverses interprétations. Dans le cas d'Hamlet, par exemple, Liszt s'appuie sur le seul titre et la référence à Shakespeare, car toute la pièce pourrait constituer le programme, ce qui en rend la distribution impossible (voir Carenco 2016, p. 293-297) ; quant à Von der Wiege bis zum Grabe, méditation sur la vie et la mort inspirée par un dessin de Zichy, on pourrait imaginer la distribution du dessin, mais les trois mouvements ont des titres qui peuvent se substituer au programme («Die Wiege " / "Le berceau ", " Der Kampf ums Dasein » / " La lutte pour la vie » et "Zum Grabe, die Wiege des zukünftigen Lebens » / "Dans la tombe, berceau de l'au-delà » ou " Berceau de la vie à venir »). Le cas de l'ultime poème symphonique de Liszt se rapproche ainsi du modèle de la Symphonie " pastorale » et d' Harold en Italie.

L'étude de la construction et la comparaison des programmes des poèmes symphoniques, qui n'ont pas été, à notre connaissance, réalisées d'un point de vue littéraire, restent des chantiers à mener. Mais concrètement, c'est la distribution de ces préfaces qui nous intéresse dans le cas de la présente étude préliminaire. Comment penser ce geste dans l'esthétique de la musique à programme?

\section{L'esthétique de la musique à programme dans les écrits de Liszt}

Outre les partitions et les paratextes musicaux eux-mêmes, de nombreux textes théoriques de Liszt donnent à penser les fonctions possibles des préfaces qu'il allie à ses partitions. On trouve dès le 12 février 1837, dans la première des Lettres du bachelier $^{13}$, un passage qui sonne a posteriori comme un véritable plaidoyer pour les paratextes musicaux que seront les préfaces des poèmes symphoniques, dans lequel le musicien n'hésite pas à tracer un parallèle avec les préfaces d'œuvres littéraires :

Il paraît peu de livres aujourd'hui qu'on ne fasse précéder d'une longue préface, qui est, en quelque sorte, un second livre sur le livre. Cette précaution, superflue à beaucoup d'égards, lorsqu'il s'agit d'un livre écrit en langue vulgaire, n'est-elle pas d'absolue nécessité, non pas à la vérité pour la musique instrumentale, telle qu'on la concevait jusqu'ici [...], mais pour les compositions de l'école moderne, aspirant généralement à devenir l'expression d'une individualité tranchée? (Liszt [1837]2000, p. 94-96)

Selon Liszt, en 1837, la nécessité de préciser le sens de l'expression musicale se fait sentir de manière d'autant plus accrue que cette expression émane d'un individu particulier. La conception $\mathrm{du}$ " genre instrumental expressif » berliozien ${ }^{14}$, teintée - dès la Symphonie fantastique - d'aspects autobiographiques, est donc bien présente

13 Ce premier texte publié avec la mention « Lettres d'un bachelier ès-musique » est adressé à George Sand sous l'expression "à un poète voyageur »; il ne doit pas être confondu avec la Lettre d'un voyageur à $M$. George Sand (publiée par Liszt dès le 6 décembre 1835) qui constitue un précurseur à la série des Lettres d'un bachelier (voir Carenco 2018).

14 Berlioz, "Aperçu sur la musique classique et la musique romantique », Le Correspondant, 22 octobre, dans Gérard et Cohen 1996, p. 63-68. 
à l'esprit de Liszt. Cela peut paraître déroutant, car la grande majorité des préfaces des poèmes symphoniques lisztiens tendront au contraire vers l'expression de grands concepts, de généralités sur l'Humanité et de considérations d'ordre philosophique sur l'art. Mais si l'on observe plus attentivement chacun des paratextes concernés, on note que, justement, les circonstances particulières dans lesquelles le compositeur a écrit la pièce sont très souvent précisées. Des sources d'inspiration sont ainsi pointées du doigt, par exemple dans les préfaces d'Orphée et de Prométhée (initialement conçues comme ouvertures), ou d'Héroïde funèbre (développée à partir de sa Symphonie révolutionnaire de jeunesse ; voir Carenco 2013 et Kaczmarczyk 2000) ; elles sont généralement multiples et filtrées à travers plusieurs couches de relectures et de références culturelles. L'exemple le plus frappant est celui du programme de Tasso, qui place l'œuvre dans le contexte des célébrations du centième anniversaire de la naissance de Goethe tout en se référant aussi largement à Byron, et qui s'appuie sur des éléments de la vie du Tasse, tout en indiquant le souvenir d'un motif mélodique entendu par Liszt à Venise. Le texte invite en fait l'auditeur à une écoute active appuyée sur une mémoire constituée à la fois d'un ensemble de références culturelles partagées et de souvenirs individuels.

Après l'avant-propos de la première édition des Années de pèlerinage (abondamment commenté par les musicologues ; voir Eigeldinger 2006 et Reynaud 2003), le texte théorique majeur sur la conception du programme chez Liszt est l'essai Sur Harold symphonie de Berlioz. Publié dans une traduction allemande en 1855 avec la signature de Liszt (Liszt 1855a), il est cependant originellement écrit en français (un manuscrit de la main de la princesse Wittgenstein est conservé à la $\mathrm{BnF}^{15}$ ). Si les tournures parfois alambiquées de ce texte en rendent la lecture difficile, il permet néanmoins d'éclaircir deux points qui sont généralement source de contradictions dans l'esthétique lisztienne de la musique à programme.

Premièrement, la question de l'antériorité du programme et du moment de sa rédaction au cours de la genèse de l'œuvre. On lit :

Le programme ne revendique que la possibilité de préciser l'émotion qui pénètre le musicien-poète lorsqu'il crée son œuvre [...]. Or, s'il est puéril, oiseux et parfois dangereux de dessiner le programme après coup, de vouloir expliquer le sentiment d'une œuvre instrumentale, d'en violer le mystère en essayant de profaner par la parole des émotions et des aspirations qui par cela même qu'elles n'ont pu se révéler que sous cette forme ne se laissent point revêtir de mots et d'images, le maître est maître de construire une œuvre sous l'empire de certaines impressions déterminées dont il désire communiquer à ses auditeurs la connaissance pleine et entière (Liszt 1855b, p. 75).

Ces lignes peuvent être troublantes lorsqu'on sait que la plupart des préfaces des poèmes symphoniques de Liszt sont effectivement rédigées dans les phases finales de

15 Manuscrit partiel de la version française originale du texte daté de 1855 (Liszt 1855b). Voir Carenco 2013, p. 85. 
la composition, voire une fois la partition achevée ${ }^{16}$. En effet, si le terme de poème symphonique apparaît dès 1854 sur une affiche de concert pour désigner une œuvre de Liszt, il n'assure pas la présence effective d'un texte de préface distribué aux auditeurs. De récents travaux (Cormac 2017) ont mis à jour la présence de l'appellation "Symphonische Dichtung » sur une affiche signalant l'interprétation des Préludes le 23 février 1854 à Weimar (figure 1), mais nous n'avons pas trouvé trace d'un texte qui aurait pu être distribué en salle à cette occasion.

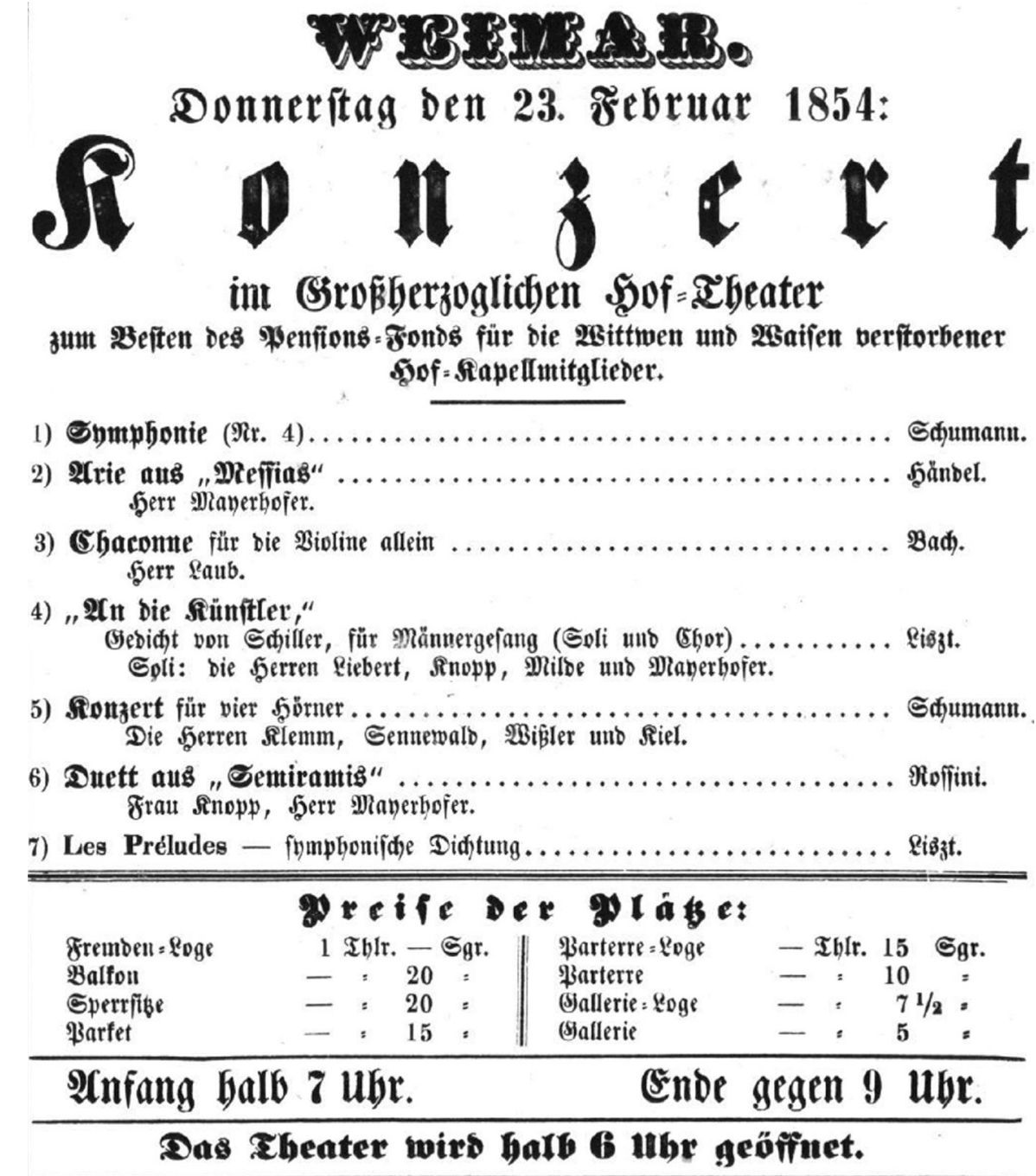

\section{Die freten Entréen zum אonzert find erít $\mathfrak{h a l b} 7$ uhgr giltig.}

Figure 1: Affichette d'un concert où se seront interprétés Les préludes de Liszt, Weimar, 23 février 1854 ; Landesarchiv Thüringen - Hauptstaatarchiv Weimar Kunst und Wissenschaft - Hofwesen, A10419/41, 91 (www.theaterzettel-weimar.de).

16 La majeure partie de la musique des poèmes symphoniques existe d'abord souvent comme ouverture (associée à une représentation d'œuvre théâtrale ou lyrique), sans aucun texte de préface. La rédaction du texte de la préface est complètement postérieure à la composition musicale au moins pour Les préludes (Cormac 2017) et Orphée (Gut 1999). 
Pourtant, même lorsque le texte de préface est formulé dans un second temps, il pointe bien des sources d'inspirations qui président à la composition, ou sont associées au processus créateur au cours de la genèse. L'aspect tardif de la rédaction des textes ne pose donc aucunement problème : il ne s'agit que d'une mise en forme ultime, qui est d'ailleurs parfois déléguée à d'autres personnes que le compositeur dans le cadre de l'atelier d'écriture de l'Altenburg (voir plus haut les exemples de paternité).

Deuxièmement, l'essai sur la symphonie Harold en 1855 permet de comprendre la difficile conciliation entre l'usage des mots et l'autonomie de la musique. Selon les conceptions exprimées dans ce texte, on ne devrait pas écouter de poème symphonique sans en avoir lu la préface, si l'on veut saisir l'œuvre dans son intégralité ${ }^{17}$. Le programme est donc indispensable, car il a pour fonction de participer à la précision de la représentation. Mais il faut veiller ici à ne pas faire de contresens, car comme l'exprime très justement Jean-François Candoni :

Liszt restait convaincu que la musique n'est pas vouée à l'imprécision par sa nature même, qu'elle n'est pas condamnée à se soumettre aux mots pour dire clairement ce qu'elle a à dire. [...] Les sentiments qu'elle exprime ne semblent obscurs que parce qu'ils atteignent un degré de différenciation et de finesse tel qu'on ne peut les traduire par des mots (Candoni 2012, p. 192).

Dans la conception lisztienne, la musique est "vague » en ce qu'elle est située au-delà des mots, et non en deçà : elle est intraduisible, car trop précise et ineffable à la fois. Les mots sont donc nécessaires pour rendre accessible la représentation. Les textes des préfaces sont ainsi destinés à éclairer à la fois la substance créatrice - niveau poiétique - et l'interprétation - niveau esthésique. Mahler parlera de l'existence d'un programme intérieur et d'un programme extérieur, idée reprise par Dahlhaus dans L'idée de la musique absolue (voir Dufetel 2018).

Conçu comme « un point d'accroche pour l'imagination » (Brendel 1859, p. 11) ou « une idée directice qui lui [l'auditeur] donne une orientation générale » (Brendel 1860, p. 76, trad. Candoni 2012, p. 206), le texte du programme n'atteint donc en aucune façon l'autonomie de l'œuvre musicale, qui est conçue comme un tout, un jeu d'interactions complexes entre le programme et la musique. Il apparaît donc réellement nécessaire, lors des exécutions des poèmes symphoniques de Liszt, de diffuser l'œuvre dans son ensemble, en distribuant le programme aux auditeurs. Que se passe-t-il concrètement dans les concerts où ces œuvres sont interprétées?

17 «Le symphoniste peintre qui prend à tâche de retracer un tableau tellement dessiné dans son esprit, une série d'émotions clairement définies et dont il se rend parfaitement compte, comment ne voudrait-il pas être parfaitement compris?» (Liszt 1855b, p. 76). 


\section{LA MÉDIATION DANS LA SALLE DE CONCERT ET LA PRESSE}

\section{Exemples de diffusion des programmes en Allemagne}

Si la France doit être considérée comme le terreau où a germé une partie de l'idée lisztienne sur la musique à programme, c'est bien en Allemagne que voient le jour ses réalisations symphoniques et les poèmes symphoniques, peu après son installation à Weimar en 1848.

La question de la médiation des programmes est problématique. Premièrement, parce que les œuvres ont été presque toujours créées dans des versions non définitives, avant la parution de la partition, et peut-être avant même que le programme ne soit rédigé. Certains poèmes symphoniques, comme Tasso ou Orphée, étaient initialement des ouvertures pour d'autres œuvres, et il semble impossible dans ce contexte de leur proposer un programme individuel : leur musique était pour ainsi dire le programme de ce qui suivait (en l'occurrence une pièce de Goethe et l'opéra de Gluck). Ainsi, nos recherches préliminaires n'ont pas révélé de traces de programmes dans la presse locale à l'occasion des premières weimariennes. Des programmes étaient-ils distribués ? Nous n'en avons pas non plus trouvé dans les archives Liszt du Goethe- und Schiller Archiv (Bestände 59 et 60), ni au Landesarchiv Thüringen - Hauptstaatsarchiv Weimar, où sont conservés les « Theaterzettel », c'est-à-dire les affichettes des concerts et des représentations théâtrales et lyriques.

Cependant, pour la première exécution de Cequ'on entend sur la montagne, le 7 janvier 1857, 1'affichette du concert comporte les lignes dont Liszt demande la reproduction (voir la figure 2). À quelques variantes typographiques près, il s'agit exactement du même texte que celui de la partition, ce qui laisse penser que les deux phénomènes de médiation (publication de la partition et concert) sont liés. D'après l'état actuel des dépouillements, c'est à la fois un cas unique et le plus ancien témoignage de médiatisation d'un programme lisztien connu dans une salle de concert ${ }^{18}$.

D'après Stefan Keym, le cas du Gewandhaus à Leipzig est aussi intéressant, car la pratique de la distribution des programmes n'y a vraisemblablement pas été d'usage avant les années $1880^{19}$. Cet exemple vaut-il pour toute l'Allemagne ou est-ce une particularité locale liée au supposé « conservatisme » du Gewandhaus, qui fut initialement réfractaire à la " musique de l'avenir »? De façon générale, les esprits et les usages du concert et de l'écoute ont peut-être été plus ouverts en France qu'en Allemagne à la pratique d'une musique accompagnée d'un texte-programme. On peut donc se demander si le prisme franco-allemand ne devrait pas être pris en compte pour l'étude de la médiation des programmes. C'est une hypothèse historique et esthétique qu'il faudra confronter à la réalité des sources et à un dépouillement systématique dépassant les limites de la présente étude préliminaire, mais l'Allemagne a peut-être

18 L'ensemble des Theaterzettel est consultable ici : http://www.theaterzettel-weimar.de (consulté le

26 novembre 2019).

19 Communication personnelle de Stefan Keym, que nous remercions. 
été moins concernée que la France, ou concernée plus tardivement, par le phénomène de diffusion de textes programmatiques. Gozlan écrit d'ailleurs que Liszt a dû publier les préfaces de peur d'être incompris, "même en Allemagne » (Gozlan 1870, p. 15 ; nous soulignons) ; c'est la France, où régnait l'art de la conversation et où se diffusaient les Paroles de concert, qui a peut-être été paradoxalement plus réceptive à cette démarche. En France, en effet, les programmes semblent arriver dès le début de la diffusion des poèmes symphoniques, à Paris comme en province.

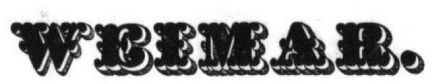

\section{Mittwod Den 7. Iamuax 1857.}

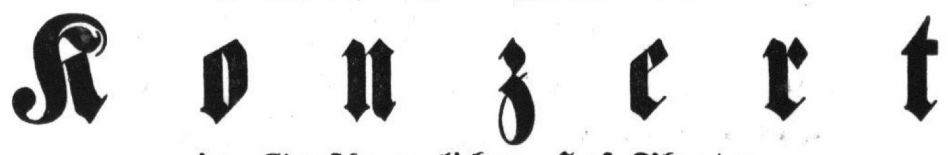

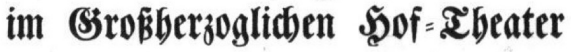

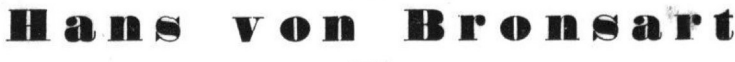 \\ zum}

Beften des Drchefter=jenfionsfonds

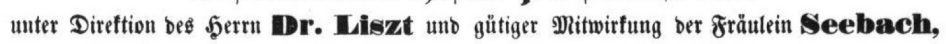

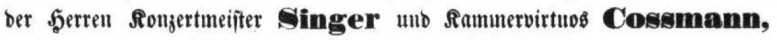

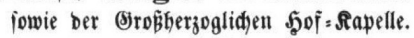

\section{PROGRAMM.}

1. Ce qu'on entend sur la montagne. Symphonifdge Didftung (nad) $\mathfrak{B}$. ફ口ugo) von Fr. Liszt.

Der Didter vernimmt gwei Stimmen; Die eine unermeglid, glorrei申 und orbnungevoll, beut פerrn ibren jubelnben Robgefang entgegenbraufent : - Die andere bumpf, voll Sdumergenslaut, von

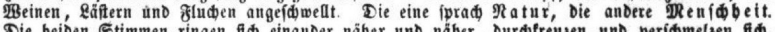

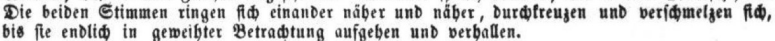

2. a) Sđb̆n Şebivig, Baflaben von 8 . Şebbel, mit Rlavierbegleitung von

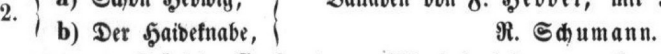
Fräulein Seebach - (Rlavierbegleitung - ફ̧err von Bronsart.)

3. Trio für ßiano, Bioline uno (Eello von Hans v. Bronsart.

a) Allegro molto, b) Scherzo-Vivace, c) Adagio funebre, d) Finale. H. v. Bronsart, $\mathfrak{j} \mathfrak{h}$. $\mathfrak{R} . \mathfrak{R}$. Singer unb $\mathfrak{R} \cdot \mathfrak{B}$. Cossmann.

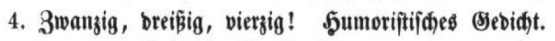
Frăulein Seebach.

5. 3weite Ronzert für \$iano mit Drdhepterbegleitung, von Fr. Liszt. H. v. Bronsart.

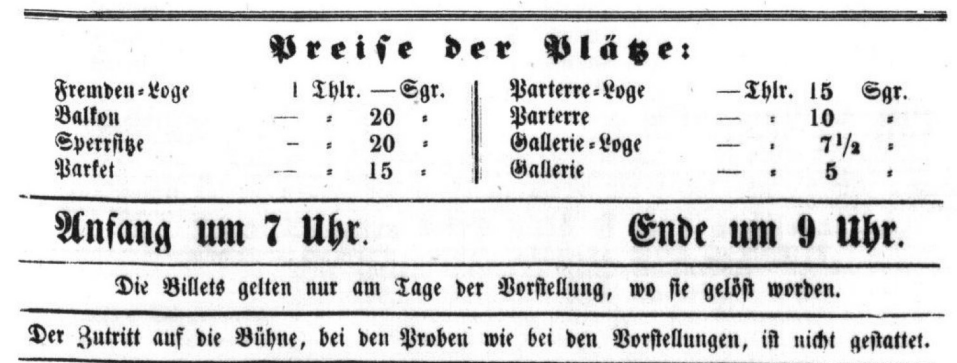

\section{Das Theater wirb un 6 ubr geärionet.}

Drud ber \$of- Budbruderel.

Figure 2: Affichette du concert de Ce qu'on entend sur la montagne de Liszt, Weimar,7 janvier 1857 ; Landesarchiv Thüringen - Hauptstaatarchiv Weimar Kunst und Wissenschaft - Hofwesen, A10419/44, 67 (www.theaterzettel-weimar.de). 
On sait que les poèmes symphoniques pénètrent en France d'abord dans leur version pour deux pianos. Le 2 mars 1860 à la salle Herz (Paris), Alfred Jaëll et Hans von Bülow interprètent Les préludes ${ }^{20}$, premier poème symphonique lisztien introduit en France. À cette occasion, dans le cadre d'une critique particulièrement acerbe signée Paul Scudo, le texte du programme est entièrement repris dans la Revue des deux mondes. Il est présenté comme "l'explication qu'en donne M. Liszt lui-même » :

Mais lorsque M. Jaell a voulu exécuter avec M. Hans de Bulow une de ces divagations à quatre mains que M. Liszt intitule Préludes symphoniques, tout le monde s'est levé, après trente ou quarante mesures, et a déserté la salle. C'est que vous ne savez pas ce que c'est que les Préludes symphoniques! Écoutez alors l'explication qu'en donne M. Liszt lui-même : " Notre vie est-elle autre chose qu'une série de préludes à ce chant inconnu dont la mort entonne la première et solennelle note ? - L'amour forme l'aurore enchantée de toute existence ; mais quelle est la destinée où les premières voluptés du bonheur ne sont point interrompues par quelque orage dont le souffle mortel dissipe ses belles illusions, dont la foudre fatale consume son autel, et quelle est l'âme cruellement blessée qui, au sortir d'une de ces tempêtes, ne cherche à reposer ses souvenirs dans le calme si doux de la vie des champs ? Cependant l'homme ne se résigne guère à goûter longtemps la bienfaisante tiédeur qui l'a d'abord charmé au sein de la nature, et lorsque la tempête a jeté le signal des alarmes, il court au poste périlleux, quelle que soit la guerre qui appelle à ses rangs, afin de retrouver dans le combat la pleine conscience de lui-même et l'entière possession de ses forces (1)?... » Voilà ce que c'est que les Préludes symphoniques, et je puis assurer que la musique est digne du commentaire qu'on vient de lire.

(1) Méditations poétiques, en tête du programme (Scudo 1860, p. 762).

Dans le cadre d'une réception vraisemblablement très difficile, le texte semble ici utilisé dans la presse pour dévaloriser encore un peu plus la partition. Quelques années après, lors de la première exécution orchestrale de l'œuvre en France le 31 octobre 1869, la préface figure sans commentaire sur le programme distribué aux auditeurs de l'Orchestre des Concerts Pasdeloup, toujours avec la même attribution (erronée) à Lamartine ${ }^{21}$.

Quelques mois plus tard, le 13 février 1870, Pasdeloup reprend Les préludes avec, entre autres, l'ouverture du Songe d'une nuit d'été de Mendelssohn. L'affichette comporte une erreur sur le titre, qui est indiqué au singulier (Prélude) - erreur ou lapsus qui souligne peut-être l'étrangeté, pour le public français, de ce titre au pluriel, et le rattache à la

20 Il s'agit certainement - d'après l'état actuel de nos recherches - de la deuxième exécution en France de la version pour deux pianos de l'œuvre (la première ayant été donnée en mars 1857 chez Érard par Hans von Bronsart et Charles Wehle). Les préludes demeure le poème symphonique de Liszt le plus souvent exécuté en France.

21 Sur la note attribuant - faussement - le texte à Lamartine, et plus globalement sur l'histoire de la rédaction de ce texte, voir Haraszti 1953 et Keym 2013. 
tradition du prélude traditionnel ${ }^{22}$. Pour ces deux œuvres, une note indique « voir au verso ", où l'on trouve en effet deux textes pour ces œuvres : des extraits du Songe de Shakespeare (acte V, sc. 2, et acte II, sc. 3) dans la traduction de Benjamin Laroche pour Mendelssohn, et la préface des Préludes, attribuée à Lamartine. La présence de textes pour ces deux œuvres révèle leurs points communs esthétiques : si le Songe n'est pas une musique à programme, ses racines littéraires sont communes avec l'œuvre de Liszt, et l'Ouverture de Mendelssohn, par l'ajout d'une préface, est assimilée à un poème symphonique. Ce geste témoigne de l'habitude croissante consistant à ajouter des programmes aux œuvres qui n'en étaient initialement pas pourvues, qu'ils s'agisse d'une projection programmatique ou d'informations historiques et analytiques dont la pratique des concerts d'aujourd'hui est l'héritière.

À l'occasion de la première parisienne de Tasso lors du Concert populaire de Pasdeloup le 3 mars 1878, le programme figure au verso (il s'agit précisément de la deuxième partie du texte de l'édition originale, consacré à l'œuvre et pas à son contexte de composition, qui n'est donc pas jugé intéressant pour l'auditeur).

Le 24 décembre 1882, la première partie de la Faust-Symphonie ("Faust ») est exécutée par Pasdeloup. Alors que Liszt ne fournit aucun programme dans cette partition, où seuls les titres font office d'éléments programmatiques, l'affichette indique des éléments ("Tristesse, Visions, Rêve de bonheur et de gloire, Déception »). Une telle liste de topoï psychologiques absents de la partition semble laisser fortement transparaître l'influence des sous-titres de la Symphonie fantastique. Les 7 et 14 janvier 1883, quand l'œuvre est reprise en entier cette fois (sans le chœur final), l'affichette comporte, au verso, un programme analytique dont l'origine ne nous est pas encore connue. En résumé, il s'agit d'ajouter un programme là où le compositeur n'en a pas prévu.

En 1886 enfin, à l'occasion d'un séjour de Liszt à Paris, quelques mois avant sa mort, Lamoureux présente Tasso le 28 mars. Le texte joint au programme est une réécriture de celui publié dans la partition, qui est cité (à la fois la partie expliquant le contexte et celle proprement sur l'œuvre). Une évolution éditoriale, un " travail » est ici à l'œuvre pour la médiation dans la salle de concert : il ne s'agit pas du texte de Liszt à la lettre, mais d'une sorte de notice qui s'en inspire et le reprend en partie. Les paratextes servent de source pour un paratexte de second niveau.

On observe aussi, toujours en 1886, la diffusion des mots de Liszt non pas dans la salle de concert, mais dans un essai d'Amédée Boutarel publié en plusieurs livraisons dans Le Ménestel au printemps (à l'occasion du séjour de Liszt à Paris). À la suite de l'interprétation d'Orphée le 28 mars 1886 aux concerts Colonne ${ }^{23}$, le commentaire du Ménestrel non seulement cite entre guillemets plusieurs phrases de la préface, mais encore reprend à son compte l'idée d'un " art civilisateur » et les

22 Les programmes (affichettes) des œuvres discutées dans les paragraphes suivants (Les préludes, Tasso, Faust-Symphonie) ont été communiqués par Yannick Simon que nous remercions chaleureusement (voir Simon 2011).

23 Ce qui semble être la première exécution orchestrale du poème symphonique en France (en l'état actuel de nos recherches). 
adjectifs de " mystérieux ", " doux ", " suave " et " mélodieux » pour les mettre en regard des principaux thèmes musicaux qu'il cite aussi (Boutarel 1886). Le ton se fait plus analytique, mais les termes de Liszt restent présents : on voit de quelle manière le discours auctorial peut orienter non seulement l'interprétation et l'écoute des partitions, mais encore les analyses que les musicologues peuvent en réaliser. Au-delà de la diffusion précise des textes dans la salle de concert, les conséquences de cette " infusion " du vocabulaire des préfaces dans la presse sont considérables sur l'appréhension que le public parisien peut avoir des œuvres. La situation est-elle similaire en province? Nous proposons de terminer cette enquête préliminaire par deux incursions ponctuelles qui montrent comment les paratextes continuent de façonner la réception des œuvres après la mort du compositeur. Les cas présentés (à Angers et à Nancy) ne prétendent pas avoir valeur de modèles par rapport à l'ensemble de la diffusion de la musique symphonique en France à cette époque ; ils offrent simplement deux exemples centrés sur le corpus des poèmes symphoniques de Liszt.

Premier exemple en province: Angers (1879-1913)

L'Association artistique d'Angers, connue plus tard sous le nom de Société des concerts populaires, a été fondée en 1877 par Alfred Michel, Jules Bordier et Louis de Romain dans un triple élan de décentralisation, de démocratisation et d'éducation. L'Allemagne en constitue le modèle absolu en raison d'une part de la vitalité de sa vie symphonique non centralisée. D'autre part, d'un point de vue proprement musical, la tradition symphonique des Classiques et des Romantiques, mais aussi Wagner, sont des modèles historiques et esthétiques qui encouragent les organisateurs angevins à soutenir le développement de l'école française (voir Dufetel 2019). C'est ainsi que Massenet, Saint-Saëns, d'Indy, Chabrier et tant d'autres sont invités à venir diriger leurs œuvres dès les premières années des Concerts populaires angevins, organisés sur le modèle de Pasdeloup (voir Simon 2006).

Les archives de l'Association, d'une immense richesse et entièrement conservées depuis plus de 140 ans, sont réparties dans différents fonds d'Angers : les partitions (conducteurs, matériel, manuscrits), après avoir été déposées au Conservatoire à rayonnement régional, sont aujourd'hui à la Bibliothèque municipale, où on trouve aussi les programmes des concerts et journaux ; les archives administratives sont conservées entre les Archives municipales et les Archives départementales de Maineet-Loire ${ }^{24}$. Presque dès sa fondation, 1'Association appuie sa saison sur la publication très régulière de périodiques sur abonnement (Angers-artiste, Angers-revue, Angers musical, etc.). À chaque concert correspond un numéro où figurent non seulement les programmes des concerts et des textes explicatifs, mais aussi des textes divers (musique, arts, vie des concerts locaux et parisiens, critiques, portraits d'artistes). La médiation, à travers ces brochures et à l'appui d'une économie éditoriale bien

24 Certaines partitions historiques sont encore conservées dans la bibliothèque de l'Orchestre national des Pays de la Loire, fondé en 1971 à partir de l'orchestre de la Société des concerts populaires. Elles sont peu à peu reversées à la Bibliothèque municipale. 
rodée, est extrêmement bien organisée : un concert est toujours précédé d'un numéro qui annonce et présente le programme, et suivi de comptes rendus dans le numéro suivant.

La Société angevine mit assez rapidement Liszt à l'honneur dans le but de proposer au public la " modernité " de l'école germanique. Le fonds comporte aujourd'hui 25 de ses partitions orchestrales et vocales, dont tous les poèmes symphoniques (à l'exception de Von der Wiege bis zum Grabe, le dernier, achevé en 1882), la FaustSymphonie, la Dante-Symphonie, Mephisto Walzer (I) et Die Legende von der heiligen Elisabeth.

Quand Les préludes sont joués pour la première fois en 1879, 1'affichette du concert reproduit le programme de la partition ${ }^{25}$. En 1901, on pouvait lire le 16 février dans Angers-artiste un extrait du programme de Tasso, qui fut joué le lendemain, introduit par ces mots : "Liszt fait précéder sa partition des lignes suivantes » (Romain 1901, p. 297).

De même qu'il avait souhaité programmer l'intégrale des symphonies de Beethoven et Mendelssohn par cycles, Louis de Romain a voulu faire connaître tous les poèmes symphoniques de Liszt au public angevin. En 1890, il se targue d'avoir donné à Angers les premières exécutions en province des Préludes, de Tasso et de Prométhée (Romain 1890, p. 307). Le tableau de la page suivante (voir la figure 3) reprend les exécutions de ses œuvres par l'orchestre de la Société des concerts populaires d'Angers qui ont été systématiquement accompagnées de quelques lignes programmatiques : soit en citant uniquement le texte de Liszt, soit en le reprenant partiellement (nous avons intégré dans le tableau toutes les œuvres symphoniques et chorales de Liszt, pas seulement les poèmes symphoniques). Cette façon de procéder se retrouve à plusieurs reprises et pour d'autres œuvres, ce qui témoigne de l'automatisme de la pratique : les programmes de Liszt sont utilisés et diffusés, en partie ou intégralement, dans les organes de la société de concerts. Quand elles n'étaient pas anonymes, les « Notices explicatives » (selon l'appellation autour de 1900) étaient principalement dues au chef Édouard Brahy et à Louis de Romain.

Les poèmes symphoniques les plus fréquemment joués furent : Les préludes : 6 fois ; Tasso et Orphée : 3 fois chacun ; Mazeppa et Hungaria : 2 fois chacun ; Festklänge et Die Ideale : 1 fois chacun. Mephisto Walzer a été jouée 4 fois et accompagnée d'un texte de nature programmatique.

Les notices angevines ont été en partie reprises dans les concerts de Nancy, initiés par Guy Ropartz qui avait des liens étroits avec ses collègues de l'Ouest. Il serait intéressant de comparer la programmation lisztienne des deux villes, non seulement pour les questions de diffusion des programmes et de réutilisation des textes, mais aussi pour voir s'il y eut des synergies de programmation, des simultanéités de calendrier voire des échanges de matériel.

25 Angers, Bibliothèque municipale, BP 21 (les programmes des premiers concerts sont collés au début des albums réunissant la collection de Angers-revue. Journal artistique et littéraire illustré). 


\begin{tabular}{|c|c|c|}
\hline Année & Date & Titre \\
\hline 1879 & 12 janvier & Les préludes (texte sur le programme) \\
\hline 1885 & 11 janvier & Tasso \\
\hline 1898 & 13 novembre & Orphée \\
\hline 1899 & 12 novembre & Les préludes \\
\hline 1900 & 7 janvier & Les préludes \\
\hline 1900 & 18 février & Faust Symphonie \\
\hline 1900 & 9 décembre & Tasso \\
\hline 1901 & 17 février & Dante Symphonie (Festival Liszt) \\
\hline 1901 & 8 décembre & Mazeppa \\
\hline 1902 & 23 janvier & Dante Symphonie (Inferno) \\
\hline 1902 & 23 novembre & Hungaria \\
\hline 1902 & 7 décembre & Mephisto Walzer (dir. Cortot) \\
\hline 1903 & 8 novembre & Les préludes \\
\hline 1904 & 9 janvier & Les préludes \\
\hline 1904 & 20 novembre & Festklänge \\
\hline 1905 & 11 novembre & Orphée \\
\hline 1906 & 18 février & Faust Symphonie \\
\hline 1906 & 9 décembre & Tasso \\
\hline 1907 & 9 mars & Mephisto Walzer \\
\hline 1908 & 22 novembre & Hungaria \\
\hline 1910 & 18 décembre & Les préludes \\
\hline 1911 & 22 octobre & Mazeppa \\
\hline 1911 & 19 novembre & Mephisto Walzer \\
\hline 1912 & 11 janvier & Mephisto Walzer \\
\hline 1911 & 17-18 décembre & Die Legende von der Heiligen Elisabeth \\
\hline 1913 & 16 mars & Die Ideale \\
\hline 1913 & 9 novembre & Orphée \\
\hline
\end{tabular}

Figure 3 : Euvres symphoniques et chorales de Liszt données à Angers par la Société des concerts populaires (18791913).

Second exemple en province: Nancy (1894-1914)

Né à Guingamp en 1864, Ropartz prend en 1894 la direction du conservatoire de Nancy et de l'orchestre qui y avait été fondé sous l'impulsion d'Édouard Brunel en 1884 (aujourd'hui connu sous le nom d'Orchestre symphonique et lyrique de Nancy). Il développe l'activité de cet ensemble installé à la salle Poirel sur le modèle de ce qu'il a observé notamment à Angers (voir Mussat 2019).

Un dossier mis en ligne sur le site Dezède par Marion Blanc et Yannick Simon répertorie les 223 concerts de l'orchestre des Concerts du conservatoire de Nancy dirigés par Ropartz entre 1894 et 1914 (Blanc 2017). Appuyé sur les collections de 
programmes conservés à Paris ${ }^{26}$, il mentionne les dates de concerts et les œuvres jouées à chaque concert, mais n'indique pas la présence ou l'absence de notices de programmes ni la nature des textes figurant éventuellement sur de telles notices. Les comptes rendus de presse de ces concerts n'ont pas non plus été systématiquement consultés. Nous avons donc mené un travail d'approfondissement pour compléter ces données grâce à la consultation de la presse locale et surtout des documents encore présents dans le fonds actuel de la bibliothèque du conservatoire de Nancy, afin d'évaluer dans quelle mesure les textes des préfaces de Liszt sont réellement disponibles pour les auditeurs de ses œuvres de musique à programme à Nancy au tournant du siècle.

\begin{tabular}{|c|c|c|c|}
\hline Année & Date & Titre $^{*}$ & Texte figurant sur le programme \\
\hline 1901 & 24 novembre & Faust & $\begin{array}{l}\text { reprise notice E. Brahy et compte-rendu } \\
\text { L. de Romain }\end{array}$ \\
\hline 1902 & 9 février & Faust & $\begin{array}{l}\text { reprise notice E. Brahy et compte-rendu } \\
\text { L. de Romain }\end{array}$ \\
\hline 1902 & 23 novembre & Dante & $\begin{array}{l}\text { commentaire de R. Pohl traduit par } \\
\text { C. Chevillard }\end{array}$ \\
\hline 1903 & 15 février & $\begin{array}{l}\text { La légende de sainte } \\
\text { Élisabeth }\end{array}$ & notice signée A.G. ${ }^{\dagger}$ \\
\hline 1903 & 25 octobre & Orphée & $\begin{array}{l}\text { reprise préface de Liszt avec } 1 \text { phrase } \\
\text { d'introduction }\end{array}$ \\
\hline 1904 & 10 janvier & Hamlet & texte explicatif ajouté, non signé \\
\hline 1905 & 12 mars & Faust & reprise notice E. Brahy \\
\hline 1908 & 20 décembre & Dante & $\begin{array}{l}\text { commentaire de R. Pohl traduit par } \\
\text { C. Chevillard }\end{array}$ \\
\hline 1909 & 14 février & La procession nocturne & traduction des vers de Lenau cités par Liszt \\
\hline 1909 & 19 février & Les préludes & reprise préface de Liszt signée \\
\hline 1909 & 24 octobre & $\begin{array}{l}\text { La légende de sainte- } \\
\text { Elizabeth [Prélude] } \\
\end{array}$ & notice d'A.G. remaniée, non signée \\
\hline 1910 & 13 février & Les préludes & reprise préface de Liszt signée \\
\hline 1911 & 29 octobre & Les préludes & reprise préface de Liszt signée \\
\hline 1911 & 12 novembre & Héroïde funèbre & $\begin{array}{l}\text { reprise préface de Liszt signée, avec une } \\
\text { introduction }\end{array}$ \\
\hline 1911 & 10 décembre & Danse macabre & reprise de propos de Calvocoressi \\
\hline 1911 & 24 décembre & Faust & reprise notice E. Brahy \\
\hline 1911 & 24 décembre & Psaume XIII & notice signée Maurice Emmanuel \\
\hline 1912 & 24 mars & $\begin{array}{l}\text { La légende de sainte } \\
\text { Élisabeth } \\
\end{array}$ & reprise notice signée A.G \\
\hline
\end{tabular}

\footnotetext{
"Nous reprenons les titres tels qu'ils figurent sur les programmes.

†' Il pourrait s'agir du musicologue Amédée Gastoué (1873-1943), membre fondateur de la Société française de musicologie. Nous remercions Jean-Christophe Branger pour cette suggestion.
}

Figure 4: Euvres symphoniques et chorales de Liszt données à Nancy par l'orchestre des Concerts du conservatoire (1894-1914).

26 Ville de Nancy, Conservatoire national de musique, Programmes des Concerts donnés à la Salle Poirel, 1894-1914, sous la direction de M. J. Guy Ropartz, Nancy, Impr. de Crépin-Leblond, 1894-1914, VMC-3727 (1894/95-1913/14). 
À la bibliothèque du conservatoire de Nancy sont conservés à la fois des partitions (matériel d'orchestre), des collections de programmes reliés par saison et, au sein d'un fonds remarquablement complet, les programmes de salle eux-mêmes, en exemplaires séparés (feuillets et livrets indépendants). Ils se présentent d'une manière moins officielle et moins institutionnalisée qu'à Angers, ce sont de simples feuillets dans un premier temps, avec la liste des titres des œuvres jouées côté recto, et de très courtes notices de programme, seulement pour certaines des œuvres, sur le verso. On constate cependant au cours de la période de plus de 30 ans une nette tendance à l'allongement des notices : dès les années 1900, on commence à trouver des programmes qui occupent des livrets de plusieurs pages.

On observe (figure 4) que plusieurs œuvres symphoniques de Liszt sont jouées sous la direction de Ropartz entre 1901 et 1912, à commencer par les symphonies Faust et Dante (respectivement quatre fois et deux fois durant cette période), et le poème symphonique Les préludes (trois fois). Parmi les poèmes symphoniques, on remarque qu'Orphée, Hamlet et Héroïde funèbre sont également interprétés respectivement en 1903, 1904 et 1911.

Des notices de programme sont imprimées et distribuées pour toutes ces œuvres, mais leur contenu varie sensiblement selon les cas : la simple réimpression des préfaces du compositeur laisse place, pour d'autres pièces, à des notices de programme rédigées de manière beaucoup plus complète, abordant des aspects historiques et analytiques. Ces notices sont souvent signées (des initiales) de leur rédacteur. Elles citent parfois des comptes rendus de presse des exécutions précédentes de l'œuvre, notamment à Angers (pour la Faust-symphonie en 1901 et 1902), et reprennent aussi parfois des ouvrages musicologiques (les propos de Calvocoressi, qui s'exprime par ailleurs sur l'habitude de rédiger des notices de programme, sont cités pour la Danse macabre en 1911 ; voir Calvocoressi 1924). Ne pouvant laisser place à une analyse détaillée de tous les types de textes associés aux œuvres musicales sur ces documents, nous nous attarderons surtout sur le traitement réservé aux textes proposés pour accompagner les poèmes symphoniques.

Comme à Angers, lorsqu'une préface de Liszt est disponible, elle est généralement reproduite sur le programme distribué en salle. C'est le cas pour Orphée, le 25 octobre 1903, avec le texte de Liszt introduit par ces mots : "Ce poème symphonique fut composé à Weimar, en Mars 1856. Voici la préface écrite par le Maître en tête de sa partition ». Le lendemain, dans le journal L'Est Républicain, le compte rendu du concert cite explicitement la préface du compositeur. Le critique évoque le caractère de sérénité lumineuse de l'œuvre, et il écrit : "C'est bien là le "flot d'harmonie" dont parle Liszt dans la préface de son œuvre » (G. B. 1903, p. 2).

Pour l'Héroïde funèbre, huit ans plus tard, le 12 novembre 1911, la préface de Liszt est reproduite sur la notice qui porte, en bas, la signature "Fr. Liszt », mais elle est précédée d'un paragraphe qui commente le texte et apporte quelques indications sur la genèse et l'œuvre :

Liszt avait écrit en 1830 une symphonie qui ne vit jamais le jour. C'est la première partie de cette symphonie, remaniée en 1849-1850, puis en 1856, qui fut publiée dans la série des Poèmes symphoniques, sous le titre Héroïde Funèbre. La copieuse préface dont l'auteur fait précéder sa partition, et qu'il est intéressant de reproduire 
intégralement, indique sous l'empire de quels sentiments avait été composée la symphonie de 1830 (Anonyme 1911).

Le lendemain, le critique musical de L'Est républicain écrit dans son compte rendu $\mathrm{du}$ concert qu'il s'agit, " en somme, [d']une musique bien adéquate à la longue et curieuse page de littérature qui lui sert de préface et que publiait le programme » (G. B. 1911, p. 2).

Les préludes sont exécutés trois fois à Nancy, d'abord en 1909, puis en 1910 et 1911, et la préface est reproduite directement dans le programme publié sur la partition du poème symphonique, trois fois également. Aucune référence à Lamartine ne figure sur ces programmes, ce qui n'empêche pas le critique de L'Est républicain au lendemain de la première exécution de l'œuvre à Nancy, le 20 décembre 1909, de mentionner que Les préludes sont « inspirés par les Méditations de Lamartine » (G. B. 1909, p. 2).

Enfin, pour Hamlet, exécuté le 10 janvier 1904 à Nancy, il n'y a pas de préface lisztienne. Les rédacteurs du programme ressentent le besoin d'écrire un texte pour accompagner l'œuvre :

En tête des partitions de ses poèmes symphoniques, Liszt place en général un argument littéraire correspondant aux divisions de l'œuvre musicale et donnant l'explication des divers épisodes. Pour Hamlet, pas de commentaire littéraire, un simple titre. Il a voulu, en effet, dans cette œuvre, évoquer le héros de Shakespeare, le plus célèbre et le plus connu de tous. Il a pensé que nul n'ignorait les traits caractéristiques de cette mélancolique figure dont il nous présente en quelque sorte le portrait musical. Nul doute que toute l'œuvre ne se rapporte exclusivement à Hamlet, à l'exception toutefois du court épisode qui deux fois intervient vers le milieu de la composition et où il est permis de trouver la vision de la douce Ophélie (Anonyme 1904).

De même que les programmes "inventés " au même moment pour la FaustSymphonie et le Mephisto Walzer à Angers, l'adjonction de ce texte explicatif montre bien de quelle manière l'exégèse est arrivée peu à peu dans le cadre de l'exécution de cette musique au début $\mathrm{du} \mathrm{xx}^{\mathrm{e}}$ siècle à être ressentie comme une nécessité. On pointe ici avec des mots le sujet que la musique illustre, offrant un « point d'accroche à l'imagination » de l'auditeur, en éprouvant le besoin d'expliquer pourquoi l'auteur n'a pas, dans ce cas particulier, laissé de préface explicative.

\section{CONCLUSION}

Le développement des recherches sur les concerts avec la mise à disposition de vastes corpus de programmes (voir par exemple dezede.org et Jardin et Taïeb 2015) permettra de faciliter l'approfondissement des questions soulevées dans notre étude préliminaire sur la médiation et la diffusion des paratextes de la musique de Liszt. Cette dernière laisse indéniablement transparaître l'évolution des pratiques du concert symphonique jusqu'à des caractéristiques que nous connaissons aujourd'hui avec le " programme de salle ». Qu'il s'agisse de citations intégrales, partielles ou de réécritures du programme du compositeur, la pensée de ce dernier innerve les salles de concert parallèlement au développement de la presse musicale. Les exemples de 
Paris, Angers et Nancy ouvrent des pistes à explorer : l'étude pourrait se poursuivre par une analyse plus poussée de la programmation complète des sociétés de concerts à Paris et dans différentes villes de province pour mettre en lumière le statut particulier que prend peut-être en France la musique symphonique de Liszt par rapport à celle d'autres compositeurs. Élargir le corpus, mais restreindre le champ géographique et temporel pourrait permettre un essai de typologisation des différentes formes d'emploi des textes des préfaces dans les programmes de salle et dans la presse musicale (du non-emploi à la citation intégrale, en passant par tous les degrés possibles de citation partielle et de réécriture).

En tout cas, il n'est pas surprenant de constater que la pratique de la notice de programme s'impose. Cependant, notre première approche permet de proposer des liens entre cette pratique et des éléments historiques et esthétiques.

Émerge tout d'abord l'idée d'une différence entre l'Allemagne et la France : il faudrait étudier si la patrie de naissance du poème symphonique a été plus lente à adopter la diffusion des paratextes que celle où Liszt avait été, jeune, au contact de l'esthétique de la musique à programme. Il semble cependant incontestable que l'habitude de distribuer un support textuel pour le poème symphonique ait libéré l'expression et la médiation au point de chercher à gloser sur des œuvres qui en sont initialement dépourvues.

La pratique du programme s'impose-t-elle à un tel point qu'elle fasse naître la nécessité d'écrire systématiquement sur toute œuvre instrumentale afin de communiquer avec l'auditeur ? Et ce particulièrement en France ? Au moins un compositeur allemand a éprouvé le besoin de passer par la rédaction d'un programme pour opérer une médiation de sa musique aux auditeurs français : Wagner. En 1860, lors de ses concerts parisiens, il rédige pour le public un texte dans sa langue pour le prélude de Lohengrin - Baudelaire le cite même dans son essai du Tannhaüser parallèlement à l'analyse de Liszt et à sa propre traduction en prose (Baudelaire [1861]1976, p. 783 et 801).

L'espace médiatique des œuvres de Liszt que nous avons exploré dans cet article correspond à une circulation complexe des paratextes entre partitions éditées, presse, brochures et autres types de médias concrets. Leur présence dans les salles de concert et dans la presse, où ils peuvent être cités en amont de l'exécution (comme support d'écoute) ou après (comme source de commentaires et comptes rendus), démontre que si le compositeur peut en contrôler la diffusion lors des exécutions qu'il supervise, elle lui échappe quand son œuvre entre véritablement dans l'espace public. D'autres s'en saisissent, mais si d'une certaine façon l'œuvre ne lui appartient plus, l'objectif du programme, sorte de "garde-fou ", est en partie accompli, car il permet de contrôler les éventuels contresens interprétatifs : si la diffusion ne l'est pas, l'écoute est en partie contrôlée. Ce lien entre paratextes et espace médiatique nous semble être un paradigme à explorer davantage dans le cadre des études de réception.

Enfin, il ne faut pas oublier que dans le cas de Liszt ces textes ne sont pas des éléments autonomes, mais qu'ils appartiennent intrinsèquement à l'œuvre, qui chez lui n'est pas que musique, mais poésie... Comme il l'écrivait avec la princesse Wittgenstein : "La réunion de la musique avec des produits littéraires ou quasi littéraires a toujours existé par le chant, mais actuellement il tend à s'établir entre elle et la littérature une 
union plus intime que jamais » (Liszt 1855b, p. 90). Oublier cet aspect serait ignorer le cœur de l'esthétique lisztienne de la musique à programme.

\section{BIBLIOGRAPHIE}

Anonyme (1830), «Épisode de la vie d'un artiste. Symphonie fantastique », Le Figaro, Ve année, n 335 (21 mai), p. 3, https://gallica.bnf.fr/ark:/12148/bpt6k2668156/f3.item, consulté le 26 novembre 2019.

Anonyme (1904), Feuillet du programme du concert du 10 janvier 1904, avec Hamlet de Franz Liszt, bibliothèque du conservatoire de Nancy.

Anonyme (1911), Feuillet du programme du concert du 12 novembre 1911, avec Hérö̈de funèbre de Franz Liszt, bibliothèque du conservatoire de Nancy.

Baudelaire, Charles ([1861]1976), "Richard Wagner et Tannhäuser à Paris », dans Euvres complètes, vol. 2, édité par Claude Pichois, Paris, Gallimard.

[Berlioz, Hector] (1830), «Grand concert », Le Figaro, Ve année, no 141 (21 mai), p. 1-2, https://gallica. bnf.fr/ark:/12148/bpt6k2666201/f1.item, consulté le 26 novembre 2019.

Berlioz, Hector ([1845]1972), Symphonie fantastique, édité par Nicholas Temperley, Kassel, Bärenreiter.

Blanc, Marion (dir.) (2017), « Les Concerts du conservatoire de Nancy sous la direction de Ropartz (1894-1914) », Dezède, https://dezede.org/dossiers/id/254, consulté le 14 juillet 2019.

Bonds, Mark Evan (1992), « Sinfonia anti-eroica. Berlioz's Harold en Italie and the Anxiety of Beethoven's Influence ", The Journal of Musicology, vol. 10, no 4 (automne), p. 417-463, https://www.jstor.org/ stable $/ 763644$ ? seq=1\#metadata info tab contents, consulté le 26 novembre 2019.

Bonner, Andrew (1986), "Liszt's Les Préludes and Les Quatre Éléments. A Reinvestigation », NineteenthCentury Music, vol. 10, nº 2, p. 95-107.

Boutarel, Amédée (1886), «L'œuvre symphonique de Franz Liszt et l'esthétique moderne », Le Ménestrel, 52 ${ }^{\mathrm{e}}$ année, $\mathrm{n}^{\circ} 18$ (4 avril), p. 137-139, https://gallica.bnf.fr/ark:/12148/bpt6k56145970/f1.item, consulté le 26 novembre 2019.

Brendel, Franz (1859), Franz Liszt als Symphoniker, Leipzig, Verlag von C. Merseburger.

Brendel, Franz (1860), "Liszt's symphonische Dichtungen "Les Préludes" und ein Urtheil von A. W. Ambros ", Anregungen für Kunst, Leben und Wissenschaft, vol. 5, Leipzig, Verlag von C. Merseburger, p. 76-78, http://opacplus.bsb-muenchen.de/title/4114502/ft/bsb10271057?page=5, consulté le 26 novembre 2019.

Calvocoressi, Michel-Dimitri (1924), "Programme-Music and Programme-Notes ", The Musical Times, vol. 65, no 971 (1 er $^{\text {Janvier}), ~ p . ~ 20-22, ~ h t t p s: / / w w w . j s t o r . o r g / s t a b l e / 911210, ~ c o n s u l t e ́ ~ l e ~}$ 26 novembre 2019.

Candoni, Jean-François (2012), Penser la musique au siècle du romantisme. Discours esthétiques dans l'Allemagne et l'Autriche du XIXe siècle, Paris, PUPS.

Carenco, Céline (2013), " De la Symphonie révolutionnaire à l'Héroìde funèbre », dans Luca Sala (dir.), "Grandeur et Finesse ». Chopin, Liszt and the Parisian Musical Scene, Turnhout, Brepols, p. 125-150.

Carenco, Céline (2013), « De l'orchestre de Berlioz au piano de Liszt. Les transcriptions d'Harold en Italie ", Revue de musicologie, tome 99, n 1, p. 79-117, https://www.jstor.org/stable/24391873, consulté le 26 novembre 2019.

Carenco, Céline (2016), «Les stratégies dramaturgiques dans Hamlet de Liszt », dans Gaëlle Loisel et Alban Ramaut (dir.), Les Comédies de Shakespeare à l'opéra (XIX'-XXI siècles), Saint-Étienne, Publications de l'Université de Saint-Étienne, p. 293-329. 
Carenco, Céline (2018), « Liszt, Franz. Lettres d'un bachelier ès-musique (1837-1841) », notice du Dictionnaire des écrits de compositeurs, https://dicteco.huma-num.fr/fr/article/2484, consulté le 6 juillet 2019.

Cormac, Joanne (2013), «From Tragedy to Melodrama. Rethinking Liszt's Hamlet», Nineteenth-Century Music Review, vol. 10, nº 1, p. 29-55, https://doi.org/10.1017/S1479409813000037, consulté le 26 novembre 2019.

Cormac, Joanne (2017), Liszt and the symphonic poem, Cambridge University Press.

Couture, Ariane (2015), " (Re)présenter les œuvres musicales. L'exemple des programmes de concert de la Société de musique contemporaine du Québec ", Revue musicale OICRM, vol. 2, $\mathrm{n}^{\circ} \quad 2$, http://revuemusicaleoicrm.org/rmo-vol2-n2/representer-les-oeuvres-musicales-lexempledes-programmes-de-concert-de-la-societe-de-musique-contemporaine-du-quebec/, consulté le 21 octobre 2019.

Doé de Maindreville, Florence (2015), "Les Paroles du concert au XVIII ${ }^{e}$ siècle. Premiers exemples de programmes de salle imprimés ", dans Étienne Jardin et Patrick Taïeb (dir.), Archives du concert. La vie musicale française à la lumière de sources inédites (XVIII ${ }^{e}$-XIXe siècle), Arles, Actes Sud, p. 31-61.

Donin, Nicolas (2011), « Pour une analyse des documents d'accompagnement du concert. L'exemple des programmes de salle du Domaine musical», dans Anne-Sylvie Barthel-Calvet (dir.), Propositions pour une historiographie critique de la création musicale après 1945, Metz, Centre de recherche universitaire lorrain d'histoire, p. 31-49.

Dufetel, Nicolas (2013), "Les écrits de Franz Liszt. Quelques réflexions épistémologiques et méthodologiques sur leur paternité et leur typologie », dans Michel Duchesneau, Valérie Dufour et Marie-Hélène Benoit-Otis (dir.), Écrits de compositeurs (1850-2000). Une autorité en questions, Paris, Vrin, p. 265-287.

Dufetel, Nicolas (2015), " Images et citations littéraires dans la musique à programme de Liszt. Pour un "renouvellement de la Musique par son alliance plus intime avec la Poésie" ", dans Alexandre Dratwicki et Agnès Terrier (dir.), actes du colloque La modernité française au temps de Berlioz (février 2010), http://www.bruzanemediabase.com/Parutions-scientifiques-en-ligne/ Articles/Dufetel-Nicolas-Images-et-citations-litteraires-dans-la-musique-a-programme-de-Lisztpour-un-renouvellement-de-la-Musique-par-son-alliance-plus-intime-avec-la-Poesie, consulté le 21 octobre 2019.

Dufetel, Nicolas (2018), «From Poiesis to Aesthesis. Imaging Programmatic Meaning in the Illustrations of Liszt's Original Editions ", dans Jonathan Kregor (dir.), Nineteenth-Century Programme Music. Creation, Negotiations, Reception, Turnhout, Brepols, p. 201-232.

Dufetel, Nicolas (2019), «La Société des concerts populaires d'Angers. Naissance, développement et contexte socio-esthétique à l'aune des écrits de Jules Bordier et Louis de Romain », dans Nicolas Dufetel et Denis Huneau (dir.), 140 ans de musique instrumentale à Angers (1877-2017). Autour de la Société des concerts populaires, actes du colloque d'Angers, UCO, 29-30 novembre 2017, Paris, L'Harmattan/Les Éditions de l'uco, 2019, p. 43-102.

Eigeldinger, Jean-Jacques (2006), " Liszt et le poème pianistique. Idées et réalisations ", dans Claude Viala (dir.), Franz Liszt pédagogue. Actes des Rencontres de Villecroze, 15-19 septembre 1999, http://doczz.fr/doc/3645175/liszt-et-le-po\%C3\%A8me-pianistique, consulté le 26 novembre 2019.

Escal, Françoise (1996), Aléas de l'ouvre musicale, Paris, Hermann.

G. B. (1903), «Conservatoire de Nancy », L’Est républicain, n 5155 (26 octobre), p. 2, https://kiosque. limedia.fr/ark:/31124/d6svc1wmlr7tzbx3/p2, consulté le 26 novembre 2019.

G. B. (1909), "Conservatoire de Nancy ", L'Est républicain, $\mathrm{n}^{\circ} 8221$ (20 décembre), p. 2, https://kiosque.limedia.fr/ark:/31124/dkpm0wn82rnwpd4f/p2, consulté le 26 novembre 2019.

G. B. (1911), «Chronique musicale », L'Est républicain, nº 8835 (13 novembre), p. 2, https://kiosque. limedia.fr/ark:/31124/d16hzbzjr1bgkpkt/p2, consulté le 26 novembre 2019.

Gérard, Yves, et H. Robert Cohen (dir.) (1996), Hector Berlioz, critique musicale, vol. 1 de La critique musicale, 1823-1863, Paris, Buchet/Chastel. 
Genette, Gérard (1987), Seuils, Paris, Seuil.

Gozlan, Louis-Léon (1870), Essai de critique musicale. F. Liszt et ses poèmes symphoniques (SymphonischeDichtungen), Marseille, E. Camoin.

Gut, Serge (1989), Franz Liszt, Paris, Fallois.

Gut, Serge (1999), «La Notion d'Art rédempteur dans Orpheus de Franz Liszt », dans Danièle Pistone et Pierre Brunel (dir.), Musiques d'Orphée, Paris, PUF, p. 115-121.

Haraszti, Émile (1953), "Genèse des préludes de Liszt qui n'ont aucun rapport avec Lamartine ", Revue de Musicologie, tome 35, $\mathrm{n}^{\circ}$ 107-108 (décembre), p. 111-140, https://www.jstor.org/stable/ pdf/927436.pdf, consulté le 26 novembre 2019.

Hoffmann, Ernst Theodor Amadeus ([1810]1985), Écrits sur la musique, traduits de l'allemand par Brigitte Hébert et Alain Montandon, Lausanne, L'Âge d'homme.

Jardin, Étienne, et Taïeb, Patrick (dir.) (2015), Archives du concert. La vie musicale française à la lumière de sources inédites (XVIIt-XIX siècle), Arles, Actes Sud.

Kaczmarczyk, Adrienne (2000), " La symphonie oubliée. Les problèmes compositionnels de la Symphonie révolutionnaire de Liszt ", Cahiers Franz Schubert, vol. 8, n 16 , p. 35-49.

Keym, Stefan (2013), « Ein Heldenleben sub specie aeternitatis? Zum Verhältnis von heroischem und religiosem Aspekt in Franz Liszts Les Préludes ", Kirchenmusikalisches Jahrbuch, vol. 97 (janvier), p. $117-132$.

Liszt, Franz ([1837]2000), "Lettre d'un bachelier ès-musique. À un poète voyageur ", Revue et gazette musicale de Paris, vol. 4 (12 février), dans Rainer Kleinertz et Serge Gut (dir.), Sämtliche Schriften, "Vol. 1. Frühe Schrifte », Leipzig, Breitkopf \& Härtel, p. 88-99.

Liszt, Franz, [et Carolyne zu Sayn-Wittgenstein] (1855a), « Hector Berlioz und seine HaroldSymphonie », Neue Zeitschrift für Musik, trad. par Richard Pohl, vol. 43, n 3 (13 juillet), p. 25-32 ; vol. $43, \mathrm{n}^{\circ} 4$ (20 juillet), p. 37-46 ; vol. 43 , no 5 (27 juillet), p. 49-55 ; vol. 43, no 8 (17 août), p. 77-84; vol. $43, n^{\circ} 9$ (24 août), p. 89-97.

Liszt, Franz, [et Carolyne zu Sayn-Wittgenstein] (1855b), Sur Harold symphonie de Berlioz, cahier manuscrit de la main de C. zu Sayn-Wittgenstein, daté et signé par Franz Liszt, Bibliothèque nationale de France, site Richelieu, ms-24359, 54 feuillets (p. 53-155).

Liszt, Franz [1857], Symphonische Dichtungen für grosses Orchester, Leipzig, Breitkopf \& Härtel, http://ks4.imslp.net/files/imglnks/usimg/8/86/IMSLP413970-PMLP41607-FLiszt Ce qu'on entend sur la montagne, S.95 fullscore firsted.pdf, consulté le 17 décebre 2019.

Liszt, Franz (1882), «Robert Schumann », dans Gesammelte Schriften, "Vol. 4. Aus den Annalen des Fortschritts. Konzert- und kammermusikalische Essays », Leipzig, Breitkopf \& Härtel, p. 103185.

Main, Alexander (1979), "Liszt After Lamartine. "Les Préludes" ", Music and Letters, vol. 60, n 2 (avril), p. 133-148, https://www.jstor.org/stable/734752?seq=1\#metadata info tab contents, consulté le 26 novembre 2019.

Mussat, Marie-Claire (2019), « La décentralisation artistique au cœur de l'imaginaire de la Troisième République dans l'Ouest de la France. Regards croisés entre Rennes et Angers, et autour du compositeur J.- Guy Ropartz ", dans Nicolas Dufetel et Denis Huneau (dir.), 140 ans de musique instrumentale à Angers (1877-2017). Autour de la Société des concerts populaires, actes du colloque d'Angers, UCO, 29-30 novembre 2017, Paris, L'Harmattan/Les Éditions de l'uco, 2019, p. 163179.

Pocknell, Pauline (2000), Franz Liszt and Agnes Street-Klindworth. A Correspondance, 1854-1886, Hillsdale, NY, Pendragon Press.

Ramaut, Alban (2018), "La fabrique de 1823-1830 ou la ur-critique d'Hector Berlioz ", Médias 19, http://www.medias19.org/index.php?id=23945, consulté le 21 octobre 2019.

Reynaud, Cécile (2003), « Regards sur la musique à programme chez Liszt et Berlioz », dans Sieghart Döhring, Arnold Jacobshagen et Gunther Braam (dir.), Berlioz, Wagner und die Deutschen, Köln, Verlag Dohr, p. 287-293. 
Romain, Louis de (1890), Essais de critique musicale, Paris, Alphonse Lemerre.

R[omain], L[ouis de] (1901), "Festival Liszt. Notice analytique - Tasso », Angers-artiste, $8^{\mathrm{e}}$ année, n 19 (16 février), p. 297, https://gallica.bnf.fr/ark:/12148/bpt6k55909068/f9.item, consulté le 26 novembre 2019.

Scudo, Paul (1860), "Revue musicale. Les concerts de la saison ", Revue des deux mondes, tome 27 (31 mai), p. 755-768, https://fr.wikisource.org/wiki/Chronique de la quinzaine - 31 mai 1860, consulté le 26 novembre 2019.

Short, Michael (2003), Liszt Letters in the Library of Congress, Hillsdale, NY, Pendragon Press.

Simon, Yannick (2006), L'Association artistique d'Angers, 1877-1893. Histoire d'une société de concerts populaires, suivie du répertoire des programmes des concerts, Paris, Société française de musicologie.

Simon, Yannick (2011), Jules Pasdeloup et les origines du concert populaire, Lyon, Symétrie.

Temperley, Nicholas (1971), "The Symphonie fantastique and Its Program ", The Musical Quarterly, vol. 57, $\mathrm{n}^{\circ} 4$ (octobre), p. 593-608, https://www.jstor.org/stable/741260?seq=1\#metadata info tab contents, consulté le 26 novembre 2019. 